\title{
Chiari I Malformation with Acute Brain Stem Compression Syndromes Requiring Emergency Neurosurgical Intervention: Report of Two Cases
}

Huan Wang*, Bonnie H Wang, Kieran P Normoyle, Arash Farahvar and William C Olivero

University of Illinois College of Medicine at Urbana-Champaign, Urbana, IL, USA

${ }^{*}$ Corresponding author: Huan Wang, University of Illinois College of Medicine at Urbana-Champaign, Urbana, IL, USA, Tel: + 217-300-1100; E-mail: huanwang@illinois.edu

Rec date: Jul 07, 2014, Acc date: Aug 14, 2014, Pub date: Aug 18, 2014

Copyright: (c) 2014 Wang $\mathrm{H}$, et al. This is an open-access article distributed under the terms of the creative Commons Attribution License, which permits unrestricted use, distribution, and reproduction in any medium, provided the original author and source are credited.

\begin{abstract}
While Chiari I malformation is a relatively common finding on MRI imaging, the vast majority of patients are asymptomatic and those who do present typically follow a slow course of chronic or subacute progression of symptoms. Emergent deterioration of existing Chiari malformation is rare. We present two patients with previously undiagnosed Chiari I malformation presenting with acute deterioration, one as a result of trauma and the other due to meningitis. Both of the patients required emergent surgical intervention, and each experienced recovery by postoperative month three. We present these two cases and review the pertinent literature.
\end{abstract}

Keywords: Chiari malformation; Trauma; Meningitis

\section{Introduction}

Chiari I malformation (CM-I) represents a congenital disorder with caudal displacement of the cerebellar tonsils through the foramen magnum into the upper cervical canal [1]. Frequently asymptomatic and incidental, it is a relatively common finding on MRI [2,3]. When symptoms present, they usually do so in a chronic or subacute fashion [4]. Rarely, acute symptoms can develop in such patients, usually related to worsening hydrocephalus, syringomyelia, benign intracranial hypertension, or rapid reduction of caudal cerebrospinal fluid (CSF) pressure[5-10]. Acute neurological deterioration has also been reported in CM-I patients after trauma from traumatic syringomyelia, penetrating injury induced spinal CSF leak, and cerebellar contusion [11-13]. We present two unique CM-I cases of severe brain stem compression symptoms developing acutely in a patient with meningitis and in another patient after a ground-level fall.

\section{Case Reports}

\section{Case 1}

This 47-year-old Caucasian female presented with one day history of headache, fever, and progressively worsening mental status. Her admission lab values were: WBC $22.32 / \mu \mathrm{l}$, CRP $87.50 \mathrm{mg} / \mathrm{L}$, and procalcitonin $55.88 \mathrm{ng} / \mathrm{ml}$. STAT MRI demonstrated CM-I without other remarkable findings (Figure 1A). Lumbar puncture was not performed and her blood culture was positive for streptoccus pneumoniae. She responded favorably to IV antibiotic treatment; however, on the third day, she developed quadriplegia and lost the gag and cough reflexes. Stat repeat MRI (Figure 1B) demonstrated downward herniation of the cerebellar tonsils $(18 \mathrm{~mm}$ below the foramen magnum). She underwent emergent Chiari decompression and recovered her brain stem reflexes and motor functions within three months.

\section{Case 2}

This 23-year-old Caucasian male had a ground-level fall witnessed by his family. He struck the back of his head against a concrete surface and, immediately afterwards, was noted to be paralyzed, unable to speak or swallow, but was able to comminute by blinking. Over the next few hours, he gradually recovered most of the neurological deficits but experienced persistent headaches, numbness/tingling in all four extremities, and swallowing difficulties. His initial neurological examination revealed no motor or sensory deficits in the extremities, but demonstrated balance/gait impairment, dysphasia with inability to handle oral secretions, and dysphonia. STAT cranial MRI evaluation demonstrated CM-I (Figure 2A). He underwent emergent Chiari decompression surgery and recovered all of his neurological deficits within three months. His three-month follow up MRI demonstrated satisfactory Chiari decompression but with persistent focal T2 signal abnormality extending from medulla to $\mathrm{C} 2$ cervical spinal cord (Figure 2B).
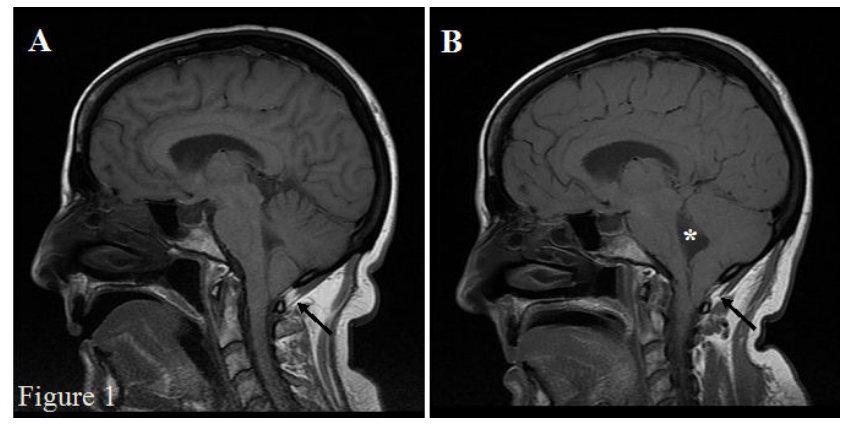

Figure 1: A) Sagittal T1 weighted MRI demonstrating CM-I (arrow) without other remarkable findings. B) Repeat MRI three days later demonstrated downward herniation of the cerebellar tonsils $18 \mathrm{~mm}$ below the foramen magnum (arrow). Also noted is distended forth ventricle (asterisk). 
Citation: Wang H, Wang BH, Normoyle KP, Farahvar A, Olivero WC (2014) Chiari I Malformation with Acute Brain Stem Compression Syndromes Requiring Emergency Neurosurgical Intervention: Report of Two Cases. J Neurol Disord 2: 177. doi: $10.4172 / 2329-6895.1000177$

Page 2 of 3
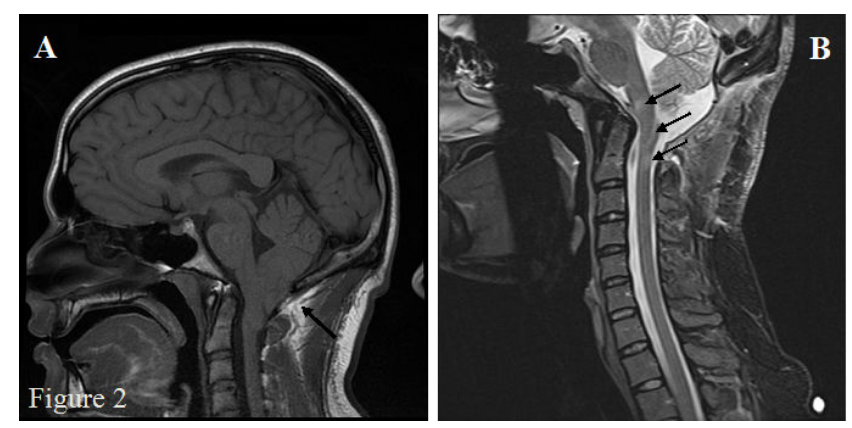

Figure 2: A) Sagittal T1 weighted MRI demonstrating CM-I (arrow) without other remarkable findings. B) Three-month follow up MRI demonstrated satisfactory Chiari decompression but with persistent focal T2 signal abnormality extending from medulla to C2 cervical spinal cord (black arrows).

\section{Discussion}

CM-I represents a congenital anatomical malformation of the posterior fossa, characterized by caudal displacement of elongated peg-like cerebellar tonsils through the foramen magnum [1]. Its exact pathogenesis and natural history remain poorly defined [14].

Although the radiographic diagnosis of CM-I remains debated $[15,16]$, the clinical consensus is that at least $5 \mathrm{~mm}$ of tonsillar descent below the foramen magnum is required to warrant such a diagnosis. Meadows et al. reviewed $>22,000$ patients undergoing MR imaging of the head and cervical spine, and reported asymptomatic CM-I to be present in $0.11 \%$ of these patients with a mean tonsillar descent $11 \mathrm{~mm}$ below the foramen magnum [2]. Vernooij et al. reviewed brain MR imaging in 2000 adults and reported that CM-I was incidentally identified in $0.9 \%$ of patients [3].

CM-I is a common presenting diagnosis for neurosurgical consultations, but the discovery is often established incidentally in asymptomatic patients. With the mean age of 25 years at presentation, most symptomatic patients report suboccipital headaches; associated symptoms include ataxia, impairment of temperature sensation, limb weakness or numbness, and clumsiness $[4,17]$. Because of the rather imprecise correlation of imaging and clinical findings and the typically insidious disease course in symptomatic patients, there are no currently well-established neurosurgical treatment recommendations and protocols for CM-I patients, especially those with no or vaguelydefined symptoms $[1,18]$.

Rarely, acute neurological deterioration in CM-I patients occurs. Sudden and dramatic worsening of symptoms have been well reported in CM-I patients due to a rapid reduction in caudal CSF pressure, most often induced following procedures such as lumbar puncture, lumbar drain placement, or lumbar-peritoneal shunt implantation [6, 19-24]. There are also isolated case reports of acute neurological deterioration due to spontaneous or penetrating spinal trauma induced decrease in caudal CSF pressure [13,25]. Conversely, ventriculoperitoneal shunt malfunction in patients with previously asymptomatic CM-I may acutely create a poorly-tolerated positive rostral CSF pressure gradient across the foramen magnum and result in foramen magnum syndrome [7,13,26,27].
Although the exact pathogenesis of syringomyelia and syringobulbia remains debated, their association with CM-I is wellestablished $[28,29]$. In some cases, acute neurological deterioration in patients with CM-I was likely due to the acute development of syringomyelia and syringobulbia $[7,12]$.

Trauma may precipitate symptoms in CM-I patients [4,27]. Rarely, devastating neurological injury and sudden death after trauma in such patients have been reported, presumably due to greatly increased susceptibility to impact-induced injury in brainstem and higher-level cervical cord at the cranial-medullary-junction in CM-I patients [11,29]. Our case 2 patient provided compelling evidence to support the notion that CM-I patients may be particularly vulnerable to serious neurological injury after trauma. However, there are no currently established neurosurgical recommendations for or against participation in contact sports in asymptomatic or minimally symptomatic CM-I patients.

Our case 1 patient acutely developed severe brainstem compression symptoms in the setting of meningitis. To date, there have been only two other similar and isolated case reports published. Hochman et al. [30] reported the acute development of dysphasia, gait impairment, truncal ataxia, decreased elevation of the palate, and a right extensor plantar response in a 37 year old CM-I female in the setting of aseptic meningitis. Jackson et al. [31] reported on a 23 year old CM-I female who presented with meningococcal meningitis and acutely developed dysphasia, right-sided hemiplegia, left-sided hemiparesis, and episodes of apnea. She underwent emergent neurosurgical intervention and recovered her neurological deficits postoperatively. CM-I patients may therefore have increased susceptibility to neurological injury at the cranial-medullary junction as a result of infection or inflammation.

\section{Conclusion}

We have presented two cases to illustrate that trauma and meningitis in the setting of CM-I can result in acute brain stem compression with potentially devastating neurological consequences. Emergent neurosurgical intervention in such cases is effective and therefore warranted.

\section{References}

1. Bindal AK, Dunsker SB, Tew JM Jr (1995) Chiari I malformation: classification and management. Neurosurgery 37: 1069-1074.

2. Meadows J, Kraut M, Guarnieri M, Haroun RI, Carson BS (2000) Asymptomatic Chiari Type I malformations identified on magnetic resonance imaging. J Neurosurg 92: 920-926.

3. Vernooij MW, Ikram MA, Tanghe HL, Vincent AJ, Hofman A, et al (2007) Incidental findings on brain MRI in the general population. N Engl J Med 357: 1821-1828.

4. Milhorat TH, Chou MW, Trinidad EM, Kula RW, Mandell M, et al. (1999) Chiari I malformation redefined: clinical and radiographic findings for 364 symptomatic patients. Neurosurgery 44: 1005-1017.

5. Chumas PD, Armstrong DC, Drake JM, Kulkarni AV, Hoffman HJ, et al. (1993) Tonsillar herniation: the rule rather than the exception after lumboperitoneal shunting in the pediatric population. J Neurosurg 78: 568-573.

6. Chumas PD, Drake JM, Del Bigio MR (1992) Death from chronic tonsillar herniation in a patient with lumboperitoneal shunt and Crouzon's disease. Br J Neurosurg 6: 595-599.

7. Elliott R, Kalhorn S, Pacione D, Weiner H, Wisoff J, Harter D (2009) Shunt malfunction causing acute neurological deterioration in 2 patients with previously asymptomatic Chiari malformation Type I. Report of two cases. Journal of neurosurgery. Pediatrics 4:170-175 
Citation: Wang H, Wang BH, Normoyle KP, Farahvar A, Olivero WC (2014) Chiari I Malformation with Acute Brain Stem Compression Syndromes Requiring Emergency Neurosurgical Intervention: Report of Two Cases. J Neurol Disord 2: 177. doi: $10.4172 / 2329-6895.1000177$

Page 3 of 3

8. Hamlat A, Helal H, Carsin-Nicol B, Brassier G, Guegan Y, et al. (2006) Acute presentation of hydromyelia in a child. Acta Neurochir (Wien) 148: 1117-1121.

9. Massimi L, Pravata E, Tamburrini G, Gaudino S, Pettorini B, Novegno F, Colosimo C, Jr., Di Rocco C (2011) Endoscopic third ventriculostomy for the management of Chiari I and related hydrocephalus: outcome and pathogenetic implications. Neurosurgery 68:950-956

10. Pettorini BL, Gao A, Rodrigues D (2011) Acute deterioration of a Chiari I malformation: an uncommon neurosurgical emergency. Child's nervous system : ChNS : official journal of the International Society for Pediatric Neurosurgery 27:857-860

11. Couldwell WT, Zhang W, Allen R, Arce D, Stillerman CB (1998) Cerebellar contusion associated with type I Chiari malformation following supratentorial head trauma: case report. Neurological research 20:93-96

12. Olivero WC, Dinh DH (1992) Chiari I malformation with traumatic syringomyelia and spontaneous resolution: case report and literature review. Neurosurgery 30: 758-760.

13. Shahlaie K, Hartman J, Utter GH, Schrot RJ (2008) Acute deterioration in occult Chiari malformation following missile spinal trauma. Case report. J Neurosurg Spine 8: 385-389.

14. Novegno F, Caldarelli M, Massa A, Chieffo D, Massimi L, et al. (2008) The natural history of the Chiari Type I anomaly. J Neurosurg Pediatr 2: 179-187.

15. Aboulezz AO, Sartor K, Geyer CA, Gado MH (1985) Position of cerebellar tonsils in the normal population and in patients with Chiari malformation: a quantitative approach with MR imaging. J Comput Assist Tomogr 9: 1033-1036.

16. Barkovich AJ, Wippold FJ, Sherman JL, Citrin CM (1986) Significance of cerebellar tonsillar position on MR. AJNR Am J Neuroradiol 7: 795-799.

17. Paul KS, Lye RH, Strang FA, Dutton J (1983) Arnold-Chiari malformation. Review of 71 cases. J Neurosurg 58: 183-187.

18. Dyste GN, Menezes AH, VanGilder JC (1989) Symptomatic Chiari malformations. An analysis of presentation, management, and long-term outcome. J Neurosurg 71: 159-168.

19. Erbay SH, O'Callaghan MG, Bhadelia R (2005) Is lumbar puncture contraindicated in patients with Chiari I malformation? AJNR Am J Neuroradiol 26: 985.
20. Hoffman HJ, Tucker WS (1976) Cephalocranial disproportion. A complication of the treatment of hydrocephalus in children. Childs Brain 2: 167-176.

21. Karabatsou K, Quigley G, Buxton N, Foy P, Mallucci C (2004) Lumboperitoneal shunts: are the complications acceptable? Acta Neurochir (Wien) 146: 1193-1197.

22. Lancione RR, Jr., Kosmorsky GS (2001) When does low mean high? Isolated cerebral ventricular increased intracranial pressure in a patient with a Chiari I malformation. Journal of neuro-ophthalmology : the official journal of the North American Neuro-Ophthalmology Society 21:118-120

23. Sullivan HC (1991) Fatal tonsillar herniation in pseudotumor cerebri. Neurology 41: 1142-1144.

24. Sullivan LP, Stears JC, Ringel SP (1988) Resolution of syringomyelia and Chiari I malformation by ventriculoatrial shunting in a patient with pseudotumor cerebri and a lumboperitoneal shunt. Neurosurgery 22: 744-747.

25. Martinot A, Hue V, Leclerc F, Vallee L, Closset M, et al. (1993) Sudden death revealing Chiari type 1 malformation in two children. Intensive Care Med 19: 73-74.

26. Levine DN (2004) The pathogenesis of syringomyelia associated with lesions at the foramen magnum: a critical review of existing theories and proposal of a new hypothesis. J Neurol Sci 220: 3-21.

27. Oldfield EH, Muraszko K, Shawker TH, Patronas NJ (1994) Pathophysiology of syringomyelia associated with Chiari I malformation of the cerebellar tonsils. Implications for diagnosis and treatment. J Neurosurg 80: 3-15.

28. Wan MJ, Nomura H, Tator CH (2008) Conversion to symptomatic Chiari I malformation after minor head or neck trauma. Neurosurgery 63: 748-753.

29. Tomaszek DE, Tyson GW, Bouldin T, Hansen AR (1984) Sudden death in a child with an occult hindbrain malformation. Ann Emerg Med 13: 136-138.

30. Hochman MS, Kobetz SA (1981) Adult Arnold-Chiari malformation type I associated with an aseptic meningeal reaction. Ann Neurol 10: 496-497.

31. Jackson RM, Penrose-Stevens A (1997) Meningococcal meningitis with Arnold-Chiari malformation. J Infect 35: 90-92. 